\title{
British Association for Paediatric Otorhinolaryngology (BAPO)
}

\author{
Abstracts from meeting 10 October 1997
}

The 7th Annual Meeting of BAPO was held at The City Hospital, Nottingham on 10 October 1997.

\section{Should GPs have Open Access Referral to ENT Operating Lists for Tonsillectomy Patients}

M. Macnamara, H. Zeitoun, M. J. Kuo, J. T. Little, W. V. Carlin.

From the Department of Otolaryngology, Head and Neck Surgery, North Staffordshire Royal Infirmary, Stoke on Trent.

This retrospective study on 237 patients referred specifically for tonsillectomy over a six-month period was designed to evaluate open access tonsillectomy referral by determining whether it constitutes good clinical practice, is popular amongst GPs and financially advantageous to GPs and/or ENT Departments. Good clinical practice was suggested by the findings that 97 per cent of referred patients had at least one sound indication for surgery with 79 per cent of these being for recurrent acute tonsillitis and 80 per cent of referred patients being actually listed. We felt that there were two important provisos. Firstly, that all children referred by this route should be screened for otitis media with effusion as this diagnosis was made in 5.5 per cent of these patients and secondly, that indications as well as indicators of severity be specifically recorded in questionnaire format to avoid the 20 per cent unnecessary tonsillectomies that might otherwise occur. Eighty-seven per cent of GPs favoured the idea of such a service with 53 per cent thinking that it would be financially beneficial to their practice. No financial gain would accrue to the ENT Department if inappropriate and additional listings are taken into account.

\section{Sleeping Position and Sudden Infant Death Syndrome: The Effect of Prone Posture on Nasal Temperature in Children}

N. Molony, C. C. Blackwell*, A. Busuttil $\dagger$

From the Department of Otolaryngology, The Royal Infirmary, Edinburgh and the Department of Medical Microbiology* and the Forensic Medicine Unit $\dagger$, University of Edinburgh, Edinburgh, UK.

\section{Background}

The incidence of sudden infant death syndrome (SIDS) has declined in response to campaigns discouraging the prone sleeping position. Recent work suggests some SIDS death may be in response to bacterial toxins produced in the upper airway. This study tests the hypothesis that the prone position raises the temperature of the upper airways in children. A temperature of $37^{\circ} \mathrm{C}$ is required for toxins to be released which could have lethal consequences in infants.

\section{Methods}

A pilot study of 10 children (aged three to eight years) and a main study of 30 children was carried out. Nasal septal temperatures were measured with an infra-red thermometer with the subjects sitting upright. Readings were then repeated after lying prone, face down for $\mathbf{3 0}$ minutes under controlled conditions of ambient temperature and humidity.

\section{Findings}

In the pilot study, mean seated temperature was $34.9^{\circ} \mathrm{C}$ (95 per cent confidence intervals (CI) $34.179-35.2^{\circ} \mathrm{C}$ ) and $36.2^{\circ} \mathrm{C}$ prone (95 per cent $\mathrm{CI}$ $\left.35.8-36.6^{\circ} \mathrm{C}\right)$. The figures for the main study were $34.53^{\circ} \mathrm{C}$ seated (95 per cent $\mathrm{CI} 34.36-35$ ) and 36.03 prone ( 5 per cent $\mathrm{CI} 35.8-36.25^{\circ} \mathrm{C}$ ). The prone readings were significantly higher in both test groups $(p<0.01)$ and the preliminary power analysis suggested the study was of sufficient size for 80 per cent power. Some subjects' prone readings were $37^{\circ} \mathrm{C}$ or higher.

\section{Interpretation}

These findings suggest that lying prone raises the upper airway surface temperature towards that required for toxin production. This supports our hypothesis of the mechanism of SIDS and could explain why prone sleeping is a risk for SIDS.

\section{Airway Management of Children with Mucopolysacharidoses Undergoing Cervical Spinal Fusion}

S. P. Hargreaves, R. Simo, D. Morris, M. P. Rothera. From the Department of Otolaryngology, Royal Manchester Children's Hospital, Manchester, UK.

The mucopolysacharidoses are a group of inherited metabolic disorders caused by a deficiency of specific lysozymal enzymes resulting in excess intracellular accumulations of partially degraded glycosaminoglycans. Because of the ubiquitous 
nature of glycosaminoglycan within the tissues of the body, a wide range of clinical effects are seen.

The anaesthetic and airway difficulties associated with this group of disorders are well known. Atlantoaxial joint instability due to hypoplasia of the odontoid process occurs particularly in the Morquio and Maroteaux-Lamy subtypes but also in Hurlers syndrome. This group of children are susceptible to acute spinal cord compression and for this reason, atlanto-axial joint fixation is advocated.

We present our experience in managing 26 of these children in collaboration with our anaesthetic and neurosurgical colleagues. Twenty children underwent pre-spinal fusion adeno/tonsillectomy. Overall three children required tracheostomy in the peri/post-operative period. Two of these were decannulated within the first seven days. The third child we have been unable to decannulate to date having developed tracheal mucopolysacharide deposits.

We advocate a very careful pre-operative assessment of the airway of these children with close monitoring especially in the early post-operative period. Adeno/tonsillectomy is usually required as part of the work up and tracheostomy may be required in the early post-operative period. Early decannulation should be a priority in this small group.

\section{Xanthoma Disseminatum - A Problem Case.}

\section{Ali, Raine.}

From the Department of Otolaryngology, Bradford Royal Infirmary, Bradford, UK

Master AM who is now 16 years old was diagnosed as having xanthoma disseminatum at the age of 11. Initial consultations were with the Department of Dermatology. The disease has gradually affected him in various ways.

1993 - Diabetes insipidus, hypothyroidism, growth hormone deficiency and temporal lobe epilepsy.

MRI scan revealed abnormality of the pituitary stalk and gland.

Skeletal survey and abdominal ultrasound were normal.

Chest X-ray was normal.

1995 - CT scan of his brain showed a lesion within the right temporal lobe.

EEG showed abnormal activity within the right temporal lobe area.

1994 - Deterioration of skin lesions. Treatment with chemotherapy initially with two courses of etoposide. Then thalidomide was tried, neither were beneficial.

1995 - Topical nitrogen mustard and later CO2 laser were applied, again with no response.

During 1995 AM developed stridor. Examination revealed numerous lesions within the oral cavity, pharynx and larynx. An aryepiglottopexy produced an initial improvement with his breathing.
1996 - Progressive stridor. Deterioration of pulmonary function. CT scan of chest was normal.

1997 - Feb. Tracheostomy was performed.

Xanthoma dissemination can affect most tissues and organs of the body. The case presents an interesting and uncommon disease. Any help with future management would be appreciated.

\section{Tracheo-oesophageal Injury in the Playground}

A. Aymat, D. W. Morgan

From the Department of Otolaryngology, Heartlands Hospital, Birmingham, UK.

We report the case of a 10 -year-old boy who fell against a wooden bench and hit the anterior part of his neck causing laceration to the trachea and oesophagus through blunt trauma to the neck. It was successfully managed without the need for tracheotomy. We present the case and review the literature over this potentially fatal injury.

\section{The Management of Epistaxis in Children with Congenital Haematological Dyscrasias}

R. Simo, F.R.C.S., D. Morris, F.R.C.S., S. Hargreaves, F.R.C.S., M. P. Rothera, F.R.C.S., R. F. Stevens, M.R.C.P.*

From the Departments of Paediatric Otolaryngology and Haematology*, Royal Manchester Children's Hospital, University of Manchester, Manchester, UK.

\section{Introduction}

The occurrence of epistaxis in the paediatric population is a very common event. The majority of the episodes originate from Little's area and are usually self-limiting or arrest with digital pressure. Just a minority require surgical cauterization in order to control these episodes. Children with congenital haematological dyscrasias present with much more severe epistaxis which potentially can be lifethreatening. These episodes require intense coagulant medical treatment, haemodynamic support and often meticulous surgical treatment in order to arrest the haemorrhage.

\section{Objective}

Analyse the clinical presentation, anatomical sites, patterns, severity, medical and surgical treatment protocol of management of children with haematological dyscrasias presenting with epistaxis.

\section{Subjects and methods}

Retrospective case-note review of 260 children with congenital bleeding disorders who have presented with epistaxis to the Otolaryngology and the Haematology Departments at the University of Manchester Children's Hospital over the past five years. The diagnoses have included congenital thrombocytopaenias, coagulation factors disorders such as haemophilia and Christmas disease, Sicklecell anaemia and von Willebrand's disease.

\section{Results and discussion}

Over a five year period, 64 (24 per cent) of 260 children registered with congenital bleeding disor- 
ders, have been identified to require acute surgical intervention to arrest epistaxis. In all of them, the epistaxis was controlled with intranasal cautery and or intranasal anterior packing under general anaesthetic, in combination with intravenous administrations of blood or plasma products and haemodynamic support. The incidence of profuse epistaxis in these children is much higher and its severity can be life-threatening. They therefore require the expert combined efforts of paediatric otolaryngologists and haematologists.

\section{Conclusions}

Epistaxis in children with bleeding disorders is much more frequent and severe than in the normal paediatric population. Combined efforts between paediatric haematologist and otolaryngologists is fundamental.

\section{Endoscopic Drainage of Paediatric Fronto- Ethmoidal Mucocoeles}

B. E. J. Hartley, V. J. Lund.

The Royal National Throat, Nose and Ear Hospital, Gray's Inn Road, London, UK.

Paediatric fronto-ethmoidal mucocoeles are extremely rare. The largest published series drained endoscopically features four cases. We present a series of seven patients drained endoscopically without complication or recurrence. The clinical presentation, radiological appearance and management is discussed. A short video demonstrating the technique of endoscopic drainage was shown.

\section{Hoarseness and Gastroesophageal Reflux in Chil- dren is There an Association?}

L. Gumpert*, N. Kalach $\dagger$, M. P. Dogliotti*, C. Dupont $\dagger$, P. Contencin*

From the Service d'ORL*, Service de Pédiatrie†, Hôpital Saint-Vincent-de-Paul, Université Paris V, Paris, France.

\section{Introduction}

In adults, gastroesophageal reflux (GOR) has been shown to play a significant role in hoarseness. However, in children, little has been published on the importance of a hoarse voice or voice change. With the exception of congenital anomalies, the aetiology is often unknown.

\section{Aim}

A prospective study to try and establish a direct association between hoarseness and GOR in children and to evaluate the type of reflux involved.

\section{Patients and method}

We present 21 children comprising 13 boys and eight girls aged between two and 14 years (mean age eight years), who had been suffering from chronic hoarseness for more than three months and had findings, on fibre-optic laryngoscopy, that were suggestive of GOR. In addition, each child had a 24-hour $\mathrm{pH}$ study. None of them complained of any gastroesophageal symptoms.

\section{Results}

Twenty-four hour $\mathrm{pH}$ monitoring revealed that 13 (62 per cent) of these children had gastroesophageal reflux and that seven ( 33 per cent) had GOR of more than three times the upper limit of normal. The $\mathrm{pH}$ graphs highlighted frequent refluxes, ranging from 0.4 to 37.4 refluxes per hour (median of 7.3 refluxes/ $\mathrm{hr}$ ). The majority of these refluxes occurred when the child was awake as opposed to asleep, with a median of 14.8 refluxes $/ \mathrm{hr}$ and 0.9 refluxes $/ \mathrm{hr}$ respectively $(p=0.002)$. The refluxes were typically of short duration.

\section{Conclusion}

This study suggests that GOR plays a direct role in the pathogenesis of hoarseness and chronic laryngitis in children.

\section{Management of Upper Airway Obstruction in Pierre Robin Sequence}

A. P. Bath, P. D. Bull

From the Department of Otolaryngology, Royal Hallamshire Hospital, Glossop Road, Sheffield, UK.

Upper airway obstruction in the Pierre Robin sequence (PRS) is potentially fatal. The vast majority of reports on the management of these infants have emanated from plastic surgeons advocating tongue to lip adhesion (TLA) procedures and condemning tracheostomy. Recent work has suggested that assessment of the upper airway with a flexible fibre-optic nasolaryngoscope would allow a more rational choice for the most appropriate procedure. Todate there have been no specific reports on the management, or outcome, of children receiving tracheostomy for relief of their upper airway obstruction in this condition.

At an institute (Sheffield Children's Hospital) specializing in paediatric airways, the preferred method of relieving upper airway obstruction in these individuals is tracheostomy. The records of all infants requiring tracheostomy for relief of airway obstruction due to PRS over a 15-year period (1982 to 1997) were reviewed.

A total of seven patients were identified. The age at which tracheostomy was performed ranged from 10 to 126 days. In all patients intubation was extremely difficult. The age at decannulation ranged from seven months (tracheostomy tube fell out at home!) to three years five months. Five infants had to undergo closure of a persistent tracheocutaneous fistula. There were no mortalities.

Experience in assessing the mechanism of airway obstruction to allow a choice of surgical intervention with such small numbers is unrealistic. Performing a tracheostomy not only offers a secure airway, but allows general anaesthetic, necessary for palatal surgery, to be given with relative safety. A TLA procedure could not guarantee these benefits. We advocate that all infants with upper airway obstruction due to PRS should be assessed by an otolaryngologist with an interest in paediatric airways with a view to performing a tracheostomy. 
Decision Making in Laryngotracheal Reconstruction and the Utility of Cricotracheal Reconstruction

J. Scott McMurray, M.D.

From the Children's Hospital Medical Center, Cincinnati, Ohio, USA.

The treatment of severe laryngotracheal stenosis in children continues to evolve. Some stenotic laryngotracheal segments are refractory to conventional expansion surgery techniques. Cricotracheal resection is now being introduced to the paediatric population. The utility and indications for this technique have not yet been completely determined. Although follow-up has been short in some of the cases in this series, partial cricotracheal resection has been performed in 26 children, from 1990 to the present. The ages were from one to 19 years. The anterior cricoid ring is removed along with the stenotic portion of the subglottis and cervical trachea. The posterior cricoid plate is then drilled flat if necessary. A suprahyoid release is performed to lower the larynx. Mobilization by anterior dissection of the cervical trachea is then performed to allow for a tension-free anastomosis to the thyroid lamina and posterior cricoid plate. The airway is managed either with intubation as a single stage procedure or with a T-tube as a stent, depending on the pulmonary status of the child. Using this technique, decannulation has been achieved in 88 per cent of children with a grade III stenosis and in 80 per cent of children with a grade IV stenosis, excluding those who have had recent surgery. Prolonged glottic oedema has made single-stage reconstruction difficult in stenoses that approach the vocal folds. The cases that seem best suited for cricotracheal resection are those with a) a severe discrete subglottic stenosis, b) a subglottic stenosis with poor cartilaginous framework support, and c) those cases with subglottic stenosis separate from the vocal folds.

\section{The Prognostic Volume of Mastoid X-Rays in Secretory Otitis Media}

Ragaie Gemaie, M.D.

From the Department of Otolaryngology, Faculty of Medicine, Cairo University, Cairo, Egypt.

The role of the mastoid air cell system in the pathogenesis of secretory otitis media has been studied previously but is still open to debate. The aim of this case-control study is to show if there is any relation between pneumatization of the mastoid and the course of secretory otitis media. Sixty-five patients (six to 10 years old) with secretory otitis media and 20 controls were selected. Patients were subjected to medical treatment and were followed up for two months. Mastoid X-rays were performed for all cases and the mastoid area of pneumatization calculated. It was noticed that patients who responded to medical treatment had a mean of 6.34 $\mathrm{cm}(\mathrm{SD} \pm 0.96)$ and a better pneumatization than those who did not respond to the same treatment who had a mean pneumatization of $4.9 \mathrm{~cm}(\mathrm{SD} \pm$
1.12). There was a significant statistical difference $(p<0.0001)$

The mean of pneumatization of the control areas was $6.89 \mathrm{~cm}(\mathrm{SD} \pm 1.14)$ with a significant difference when compared to non-responders $(p<0.0001)$, and no statistical significance when compared to patients who responded to medical treatment $(p>0.05)$.

\section{Dry Tap at Myringotomy: A Three Year Study of 1688 Children Undergoing Myringotomy}

A. H. Jardine, A. R. Maw, S. Coulton

From the Department of Otolaryngology, Bristol Royal Hospital for Sick Children, Bristol, UK.

\section{Aim}

To determine the prevalence of 'dry tap' at myringotomy and the factors associated with it.

\section{Introduction}

The reported prevalence of 'dry tap' at myringotomy varies between 0 per cent (Dempster et al., 1993) and 34 per cent (Black et al., 1990). Possible causes include resolution of glue ear and the effect of nitrous oxide anaesthesia. Establishing the prevalence and reasons might help to prevent unnecessary surgery.

\section{Method}

Children undergoing myringotomy over a three year period were included. Data was collated and analysed with Statistica. Analysis of dry taps by hospital, waiting-list time, hearing loss and tympanogram at listing, differences between first and second operated ear, age and sex of the child and season was made. In a subgroup of patients clinicians stated the main reason for listing as chronic OME or recurrent acute otitis media.

\section{Results}

Of 1688 children undergoing myringotomy the percentage of bilateral dry taps was 15.9 per cent. The dry tap rate was significantly higher in the following groups: older children, a tympanogram at the time of listing of $\mathrm{A} \mathrm{C1}$ or $\mathrm{C} 2$, milder hearing loss, recurrent acute otitis media and operation during summer or autumn. There was no significant relationship between the dry tap rate and the time on the waiting list, the sex of the child, or the grade of operating surgeon. There was no difference between the dry tap rate in the first and second operated ear in 614 children (the first year of the study).

\section{Conclusion}

The reasons for a dry tap at myringotomy are the same as those known to be associated with resolution of OME. Careful pre-operative, reassessment of any child with a Type A C1 or C2 tympanogram at the time of listing should be made to prevent unnecessary surgery. Separating those with recurrent acute otitis media who require grommets regardless of fluid at myringotomy from those with OME would prevent these being included in the overall dry tap rate. 


\section{References}

Black, N. A., Sanderson, C. F., Freeland, A. P., Vessey, M. P. (1990) A randomized controlled trial of surgery for glue ear. British Medical Journal 300: 1551-1556.

Dempster, J. H., Browning, M. D., Gatehouse, S. G. (1993) A randomized study of the surgical management of children with persistent otitis media with effusion associated with a hearing impairment. Journal of Laryngology and Otology 107: $284-289$

\section{Measures of Health, Behaviour and Quality of Life in Otitis Media with Effusion (OME)}

\section{S. Smith, M. Gannon, S. Hind, M. Haggard}

From the MRC Institute of Hearing Research, Nottingham, UK.

For young children, measures of health, quality of life (QOL) and behaviour are largely limited to parental report. Few standardized measures of health and QoL exist; although several standardized behaviour measures have been developed these are mainly either screening measures or oriented towards pathological extremes that will be rare in a mild condition. In designing the TARGET trial for treatments in OME it was therefore necessary to develop new outcome measures that would be sensitive to changes near the mean. To address both relevance and representativeness, the large numbers of children necessary to develop scores come both from the trial itself and its population control sample. We have derived scores that measure these three concepts, some of which are brief enough for routine clinical monitoring or incorporation in clinical research where the time parents can give is short.

The derivation procedure starts by establishing a pool of items found useful clinically or in other studies, though the wording often has to be amended to achieve a common format. Some entirely new items have also to be generated, particularly for the TARGET behaviour measure. The next stage is piloting of format to maximize appropriateness for the particular type of respondent and non-extremeness of the response distribution. The final three stages are the demonstration of reliability, internal consistency and three types of validity: (1) discriminant validity (for TARGET the separation is maximized between the otitis media with effusion (OME) clinical group and population controls); (2) responsiveness to change, (both developmental change and OME remission or treatment effects); and (3) external validity (construct or concurrent). For TARGET outcomes, work on (1) and (2) is largely complete; some studies on (3) are still in hand. These stages throw out of the pool many items, including some of apparent 'face' validity, and ensure that the resulting scores display requisite psychometric properties and use clinical time efficiently. Paediatric ORL is now in a position to use sets of questions with known response profiles and percentiles of the normal population distribution for children aged four to seven, in four areas likely to be of clinical interest: (1) OM-related behaviour (16 items), (2) Ear symptoms (six items), (3) URTI symptoms (five items), (4) General health (five items) for evaluation purposes in trials or audit studies or quantifying severity at assessment.

We acknowledge the MRC OM study group's assistance in providing TARGET data, and Bristol, Glasgow and East Midlands schools in providing population control data.

\section{Selective IgA Maturational Immunodeficiency and the 'Otitis-Prone' Child}

Peter J. Robb, B.Sc., F.R.C.S., F.R.C.S.(Ed.), K. Gowrinath, M.S., F.C.R.S.

From the Department of Otolaryngology, Epsom Healthcare NHS Trust, Epsom, Surrey, UK.

The otitis-prone child is well recognized in the age group much younger than those generally presenting with otitis media with effusion (OME). The toddler with repeated attacks of acute otitis media usually presents in the first year of life, and has passed the phase of recurrent acute otitis media (AOM), by three to four years of age.

During the 27 months up until February 1996, 364 children, four years old or younger, were seen as new referrals. Of these children, 170 had a primary complaint of recurrent AOM, defined for the purposes of this investigation as six or more attacks per year. The sex ratio was 2.3 males to one female.

Associated symptoms at presentation included hearing loss (39 per cent), speech and language delay and pronunciation difficulties (21 per cent), repeated perforation of the tympanic membrane (10 per cent) and other associated infective problems including infantile brochiolitis (eight per cent).

Initial management was low-dose prophylactic antibiotic treatment with trimethoprim elixir at a half normal $\mathrm{mg} / \mathrm{kg}$ dose on a once daily schedule: Forty children ( 24 per cent), failed medical management and came to surgery for insertion of ventilation tubes. With parental consent, blood was sent for immunoglobulin estimation at the time of surgery from these children.

Seventy five per cent of the surgical group were found to have an abnormally low serum IgA concentration compared with expected age corrected levels. No pattern of IgG or IgM deficiency was noted. Of the abnormal IgA levels, 42.5 per cent occurred in the one to two year age group.

We report an important association between selective IgA immunodeficiency and recurrent AOM in pre-school children. It is proposed that this is a maturational deficiency of infancy and early childhood. The implications for further investigation and treament are discussed.

\section{Necessity of Hearing Disability Measures in Otitis Media Effusion (OME)}

J. A. Birkin*, D.A. Adams $\dagger$, R.J. Flanagan $\dagger$, J. M. Higson*

From the MRC Institute of Hearing Research*, Nottingham and the Department of Otolaryngology $\dagger$, Queen's University, Belfast, UK.

Concerns voiced by parents of children with otitis media with effusion (OME) sometimes suggest that 
the degree of disability the child experiences was more severe than the results of conventional testing methods on a single day would indicate. The degree of disability is only moderately well predicted by conventional audiological tests and parental report is not referenced to norms. In order to assess the disability associated with OME, we have measured children's ability to discriminate speech-in-noise at a signal-to-noise ratio $(\mathrm{S} / \mathrm{N})$ at reception threshold, and compared this to the results of conventional tests.

The children studied $3.25-6.75$ years, with OME (but no previous ear surgery or adenoidectomy) were assessed using tympanometry, audiometry (warble tone), and full clinical examination. The IHR-McCormick Automated Toy Test was used to assess the ability to discriminate speech-in-noise. The study is longitudinal with children receiving a series of visits spanning 15 months. A control population of children completely free of an ear problem history ('normals') is being obtained. This paper reports the findings at the first visit to ENT.

A previous paper described initial results showing that the mean $S / N$ value for speech-in-noise in children with $\mathrm{B}+\mathrm{B}$ or $\mathrm{B}+\mathrm{C}_{2}$ tympanograms plus a better ear average threshold (BEAV) of $\geqslant 20 \mathrm{dBHL}$, was poorer than that of children with the same tympanogram combinations but BEAV $<20 \mathrm{dBHL}$. We have now examined S/N values systematically for differing tympanometric groups (Jerger classification) treating hearing as a separate variable. A significant difference was found between children with B + B tympanograms and other tympanogram types; however, no significant differences were found among the latter types, for which the majority of cases lay within the range shown for normal children. This indicates that in the absence of audiometry between $\mathrm{B}+\mathrm{B}$ and the rest, any cut-off should fall. Regression equations for $S / N$ showed that in the region of 15 to $25 \mathrm{dBHL}$ a combination of $\mathrm{BEAV}$ and tympanogram does not accurately predict the degree of disability. Speech-in-noise tests may be justified to better define the severity of the impact of OME in such cases, particularly as the results reflect past history as well as status of the ear on test day.

The results from further stages of this investigation will show if the addition of speech-in-noise information helps to select children who would benefit from treatment, and possibly provide a measure of the degree of benefit that a child has received from a treatment regime.

\section{Childhood Chronic Suppurative Otitis Media (CSOM) - Results of Surgery}

\section{S. B. Nair, S. Carrie*, P. J. D. Dawes*}

From the Departments of Otolaryngology, North Riding Infirmary Middlesborough, and City Hospitals, Sunderland*, UK.

We report on the results from a prospectively collected series under the care of a single consultant. Twenty-eight children underwent treatment for cholesteatoma (17) or for active retraction pocket disease (11). Three and two patients respectively were lost to follow-up.

Of those attending follow-up 11 underwent open cavity surgery for cholesteatoma and three children had similar surgery for their retraction pocket. The remaining nine had tympanoplasty.

Overall at six months 95 per cent of ears were dry and 91 per cent at 12 months. The discharging ear at six months was from a mastoid cavity and by 12 months one of the atticotomies was discharging because of a perforation following acute otitis media.

Seven of the tympanoplasties had a primary ossiculoplasty of which six either maintained or improved their hearing thresholds. The Glasgow Benefit Plots will be presented for both mastoidectomy and tympanoplasty.

\section{The Evidence that the Short-Term Resolution Rate of Otitis Media with Effusion (OME) can be Increased with Antibiotics - the Effect of Sample Size}

W. Colquhoun-Flannery, C. J. Randall, J. A. S Carruth.

From the Department of Otolaryngology-Head and Neck Surgery, Southampton University Hospital, Southampton, UK.

\section{Introduction}

The received wisdom is that antibiotics have little or no part to play in the management of otitis media with effusion (OME). The real effect of antibiotics on short-term resolution rates in OME however, is obscured by numerous, poorly-designed trials in the literature.

We examined the data of 12 trials of OME treated with antibiotics. The trials were chosen because they had been previously identified, in the literature, as having adequate trial design. Data were extracted from the individual studies and tabulated.

\section{Results}

The sample odds ratio, confidence interval, sample size required to reach significance and chi-squared statistic were calculated for each study. These are shown over (Table 1).

Because the whole population was not sampled, there was, of course, some variation in the sample odds ratios: to discover if this represented true differences in the population, the confidence interval was calculated. Confidence intervals containing 1 (i.e., suggesting antibiotic and control groups were equal) were interpreted as not significant. Significance tested this way was. compared with the chisquared test.

\section{Discussion}

Only three studies had odds ratios that did not contain one (Healy, 1984; Ernstson and Anari, 1985; Cantekin et al., 1991). This significance was confirmed with the chi-squared test. The interesting point is that all three had adequate sample sizes. DeCastro could not be analysed because none of the control effusions resolved spontaneously (giving a 
TABLE I

RESULTS

\begin{tabular}{|c|c|c|c|c|c|c|}
\hline Study & & Odds ratio & $\begin{array}{c}\text { Confidence } \\
\text { limits }\end{array}$ & $\begin{array}{c}\text { Total } \\
\text { sample size } \\
\text { achieved }\end{array}$ & $\begin{array}{l}\text { Total } \\
\text { sample size } \\
\text { required }\end{array}$ & $\begin{array}{l}\text { Chi-squared } \\
\text { significance }\end{array}$ \\
\hline Cantekin et al. & & 2.11 & $1.12-4.01$ & 518 & 598 & $<0.05$ \\
\hline Ernston and Anari & & 8.73 & $2.92-26.09$ & 91 & 32 & $<0.001$ \\
\hline Healy & & 19.94 & $7.99-49.78$ & 200 & 18 & $<0.001$ \\
\hline deCastro et al. & & - & - & 30 & 30 & $?$ \\
\hline Moller and Dingsor & $\therefore$ & 0.59 & $0.26-1.33$ & 141 & 658 & NS \\
\hline Schloss et al. & & 0.75 & $0.22-2.56$ & 54 & 1996 & NS \\
\hline Varsano et al. & & 1.11 & $0.29-4.21$ & 35 & 11390 & NS \\
\hline Mandel et al. & & 1.16 & $0.65-2.07$ & 331 & 7204 & NS \\
\hline Corwin et al. & & 1.86 & $0.95-3.65$ & 149 & 352 & NS \\
\hline Schwartz and Rodriguez & & 1.90 & $0.73-4.95$ & 69 & 304 & NS \\
\hline Giebink et al. & & 2.17 & $0.59-7.99$ & 39 & 216 & NS \\
\hline Daly et al. & & 6.25 & $0.66-59.03$ & 42 & 98 & NS \\
\hline
\end{tabular}

NS = not significant.

The odds ratio is the ratio of responders to non-responders in the antibiotic group divided by the ratio of responders to nonresponders in the control. If the groups were equal, the odds ratio would be 1.

division by zero error). One can only speculate about the other eight trials.

If the 12 trials represent the best evidence available, we seem to be treating OME on the evidence of the eight trials that did not reach significance and which provide no evidence at all. If the eight had had adequate sample sizes, we might, now, all be using antibiotics as the first line treatment for OME.

\section{References}

Cantekin, E. I., McGuire, T. W., Griffith, T. L. (1991) Antimicrobial therapy for otitis media with effusion ('secretory' otitis media). Journal of the American Medical Association 266: 3309-3317.

Corwin, M. J., Weiner, L. B., Daniels, D. (1986) Efficacy of oral antibiotics for the treatment of persistent otitis media with effusion. International Journal of Pediatric Otorhinolaryngology 11: 109-112.

Daly, K., Giebink, G. S., Lindgren, B., Anderson, R. S. (1980) Controlled clinical trial for prevention of chronic otitis media with effusion. Proceedings of the Fourth International Symposium on Recent Advances in Otitis Media June 1-4 1987; Bal Harbour (FL), BC Decker, Toronto.

DeCastro, F. J., Jaeger, R. W., Martin, L., Terneck, J. W., Tournour, B. (1982) Serous otitis media: a double-blind trial with sulfisoxazole, Modern Medicine 79: 629-630.

Ernstson, S., Anari, M. (1985) Cefaclor in the treatment of otitis media with effusion. Acta Otolaryngologica (Suppl 424): 17-21.

Giebink, G. S., Batalden, P. B., Le, C. T., Lassman, F. M., Buran, D. J., Seltz, A. E. (1990) A controlled trial comparing three treatments for otitis media with effusion. Pediatric Infectious Diseases Journal 9: 33-40.

Healy, G. B. (1984) Antimicrobial therapy of chronic otitis media with effusion. International Journal of Pediatric Otorhinolaryngology 8: 13-17.

Mandel, E. M., Rockette, H. E., Paradise, J. L., Bluestone, C. D., Nozza, R. J. (1991) Comparative efficacy of erythromycin-sulfisoxazole, cefaclor amoxicillin or placebo for otitis media with effusion in children. Pediatric Infectious Diseases Journal 10: 899-906.

Moller, P., Dingsor, G. (1990) Otitis media with effusion: can erythromycin reduce the need for ventilating tubes? Journal of Laryngology and Otology 104: 200-202.

Schloss, M. D., Dempsey, E. E., Rishikof, E., Sorger, S., Grace, M. G. A. (1987) Double blind study comparing erythromycin-sulfisoxzole (Pediazole) t.i.d. to placebo in chronic otitis media with effusion. In Proceedings of the Fourth International Symposium on Recent Advances in Otitis Media, BC Decker, Toronto.
Schwartz, R. H., Rodriguez, W. J. (1982) Trimethoprimsulfamethoxazole treatment of persistent otitis media with effusion. Pediatric Infectious Diseases Journal 1: 333-335.

Varsano, I., Volovitz, B., Mimouni, F. (1985) Sulfisoxazole prophylaxis of middle ear effusion and recurrent acute otitis media. American Journal of Disease Control 139: 632-635.

Williams, R. L., Chalmers, T. C., Stange, K. C., Chalmers, F. T., Bowlin, S. J. (1993) Use of antibiotics in preventing recurrent acute otitis media and in treating otitis media with effusion. Journal of the American Medical Association 270(11): 1344-1351.

\section{The Extended Sistrunk Procedure for Thyroglossal Ducts, Cysts and Sinuses}

N. N. Patel, B. Hartley, D. J. Howard

From the Professional Unit, Royal National Throat, Nose and Ear Hospital, London, UK.

Recurrent disease continues to plague otorhinolaryngologists, general surgeons and paediatric surgeons alike in their management of thyroglossal duct remnants. However, there are few better examples in surgery where applied anatomy and embryology proffer a solution to a problem than in definitive surgery for thyroglossal ducts, cysts and sinuses. Extending the Sistrunk operation with an en bloc anterior dissection allows confident removal of the entire thyroglossal tract remnant and thus overcomes the problem of recurrence. We discuss operative technique and scientific rationale. Review of our series demonstrates that there is no increased morbidity and indeed results would recommend adoption of the procedure.

\section{Catch 22 - A Challenge to the Otolaryngologist}

L. Ramamurthy

From the Department of Otolaryngology, Withington Hospital, Manchester.

Velocardiofacial syndrome (VCFS) comprises of cleft palate (overt/occult), cardiac defect and typical facial features. It was described by Shprintzen (1978) in 12 children. Over 150 cases have been reported in the world literature. 
As an otolaryngologist, it is important to be familiar with the features of the syndrome as many of these children have occult submucous cleft palate with velopharyngeal insufficiency. They are also prone to recurrent otitis media with effusion. The facial appearance is similar to 'adenoid facies'. If adenoidectomy is performed it can lead to further hypernasality.
We report the clinical finding in 14 patients with this syndrome highlighting the facial anomaly, palatal, audiological and otological findings. Six patients had adenoidectomy, five for recurrent effusion and one for 'nasal speech' leading to further incompetence of the velopharyngeal sphincter.

The aim of this paper is to emphasize the salient features of the syndrome so as to avoid unnecessary surgical complications. 